\title{
MODELLING CHANGES IN THE DIELECTRIC AND SCATTERING PROPERTIES OF YOUNG SNOW-COVERED SEA ICE AT GHz FREQUENCIES
}

\author{
By MARK R. Drinkwater* and G.B. Crocker
}

(Scott Polar Research Institute, University of Cambridge, Lensfield Road,

Cambridge CB2 1ER, England)

\begin{abstract}
Observations of the physical properties of the snow cover and underlying young fast ice in Resolute Passage, N.W.T., Canada, were made during the winter of 1982. Detailed measurements of snow density, and ice and snow temperatures, salinities, and brine volumes were made over a period of $46 \mathrm{~d}$, beginning when the ice was $0.4 \mathrm{~m}$ thick and about $8 \mathrm{~d}$ old. The recorded values are used in a theoretical mixture model to predict the dielectric properties of the snow cover over the microwave frequency range. The results of this analysis are then used to investigate the effects of the snow properties on the radar back-scatter signatures of young sea ice. The results show that back-scatter is a function of the incidence angle and can change significantly over short periods of time during the early evolutionary phase of ice and snow-cover development. This has important consequences for the identification of young ice forms from Synthetic Aperture or Side Looking Airborne Radar images.
\end{abstract}

\section{INTRODUCTION}

The surface of young sea ice is covered by a thin, highly saline layer of slush. The presence of this moist surface layer is frequently mentioned in the journals of early explorers and scientists working in both Arctic (Vrangel', 1840; De Long, 1883; Greely, 1886; Collinson, 1889; Malmgren, 1927) and Antarctic (Scott, 1905; Arctowski, 1908; Mawson, 1909; Wright and Priestley, 1922) regions, and has been known to the indigenous peoples of the Arctic for centuries (Nelson, 1969). However, references to such a surface layer in modern scientific literature are rare, largely due to the standard technique of measuring sea-ice salinity profiles, whereby cores are cut into $0.05-0.10 \mathrm{~m}$ sections and fail to show the finer detail of the profile near the surface (Martin, 1979), and to the general paucity of field studies on thin ice during winter. Martin (1979) measured salinities on newly formed sea ice in the Beaufort Sea as high as $95 \%$, while Crocker (unpublished) found salinities on fast ice in Resolute Passage, N.W.T., as high as 110\%, and on leads in the Arctic Ocean up to $150 \%$. As the ice thickens, frost flowers (a form of hoar frost) begin to appear on its surface and, gradually increasing in size and number, can develop into a continuous snow cover without mass inputs from blowing or falling snow. The frost flowers contain considerable quantities of salt and through capillary suction draw additional brine from the surface, resulting in a highly saline snow cover (particularly in the near-surface layers) containing large amounts of liquid even at very low temperatures. Normally, within 1 or 2 weeks of formation, the structure of the frost-flower layer is modified by strong

*Present address: Jet Propulsion Laboratory, Ms 300-323, California Institute of Technology, Pasadena, California 91109, U.S.A. winds, of ten accompanied by falling or drifting snow, and constructive and destructive metamorphosis, but it maintains some of its original characteristics, most importantly the high brine volume.

Although comprising only a few centimetres, this moist snow layer is by no means insignificant. The wetness and high salinity affect heat and vapour transfers (Ono and Kasai, 1985), the reflectivity and emissivity of the ice (Ramseier and others, 1975; Grenfell, 1986; Lohanick and Grenfell, 1986), and brine drainage and air-bubble patterns during spring melting (Martin, 1979). Despite this, measurements of snow-cover salinities are rare. Spot measurements have been reported by Martin (1979), and some time-series data can be found in Lapp and Prashker (unpublished) and Takizawa (1985), but to the authors' knowledge the only detailed study of the spatial and temporal variations in snow-cover properties, including brine volume, is that of Crocker (unpublished). The influence of the brine on the thermal conductivity of snow has been modelled by Crocker (1984), but the presence of brinesoaked snow is also of direct significance to active microwave radar remote-sensing techniques. These data therefore provide us with an opportunity to investigate the dielectric and scattering properties of such surfaces. In the following sections, we present a simple theoretical analysis of the effect of brine on the complex dielectrics of a shallow snow cover, focussing on the effects of temporal variations in the physical and electromagnetic properties of the surface layers upon scattering of incident energy over the microwave range of frequencies. The implications of these changing snow properties for active microwave remote sensing of ice characteristics are investigated.

\section{SNOW-COVER PROPERTIES}

The snow-cover data used in this paper are from Crocker (unpublished) and have been summarized in Crocker and Lewis (1985). The measurements were made on young sea ice in Resolute Passage, N.W.T., during November and December 1982. Daily averages of snow temperature, density, salinity, and brine volume were computed at $1 \mathrm{~cm}$ depth intervals from nine samples taken from a $3 \times 3$ grid on a uniform fast-ice sheet. Temperatures were measured with a calibrated Fenwal GB32P2 glass probe thermistor and Data Precision model 245 digital multimeter. A purposebuilt snow sampler was used to collect known volumes of snow from each layer, which were bagged and returned to Resolute for weighing, and the salinity of the melt determined with a YSI model 32 conductivity meter calibrated against standard ocean water. The ice first began to form on 7 November, and detailed measurements were started on 15 November when the ice was $0.40 \mathrm{~m}$ thick and the frost-flower cover had grown sufficiently to be considered a continuous snow cover. Although some measurements of the ice-surface and frost-flower salinities, and the frost-flower density, were made during the first 
week of ice growth, difficulties in sampling and interpreting the data make them of limited use in the current study. Therefore, only data from 15 November onward will be used here, and the analysis should be considered applicable only to periods when a continuous, frost-flower-based snow cover is present.

From the snow temperature, density, and salinity, the volume fractions of air, pure ice, and brine (the quantities of interest in modelling the snow-cover dielectric properties) can be calculated from the phase relations for sea ice. After measuring the salinity of a melted snow sample and subtracting the proportion of salt which will be in a solid form (the cryohydrates) at the measured snow temperature (see Assur, 1960), the brine volume of the salt/ice mixture can be determined from tables (Assur, 1960) or equations (Frankenstein and Garner, 1967). As the densities of brine and ice are different, the brine volume determined in this manner cannot be directly converted to the volume fraction of brine in the snow-pack. First, the weight ratios of brine $\left(R_{\mathrm{b}}\right)$ and ice $\left(R_{\mathrm{i}}\right)$ must be calculated

$$
\begin{aligned}
& R_{\mathrm{b}}=\frac{v_{\mathrm{b}} \rho_{\mathrm{b}}}{\left(1-v_{\mathrm{b}}\right) \rho_{\mathrm{i}}+v_{\mathrm{b}} \rho_{\mathrm{b}}}, \\
& R_{\mathrm{i}}=\frac{\left(1-v_{\mathrm{b}}\right) \rho_{\mathrm{i}}}{\left(1-v_{\mathrm{b}}\right) \rho_{\mathrm{i}}+v_{\mathrm{b}} \rho_{\mathrm{b}}}
\end{aligned}
$$

where $\rho_{\mathrm{i}}$ is the density of pure ice, and $\rho_{\mathrm{b}}$ is the density of brine, approximated using

$$
\rho_{\mathrm{b}}=\rho_{0}(1+\gamma T)
$$

where $\rho_{0}$ is the density of pure water, $\gamma$ is a constant (roughly $10^{-2}$ ), and $T$ is the temperature in degrees Celsius (Eide and Martin, 1975). The mass of each component is found by multiplying its weight ratio by the snow density $\left(\rho_{\mathrm{S}}\right)$, and the true volume fractions of brine $\left(V_{\mathrm{b}}\right)$ and ice $\left(V_{\mathrm{i}}\right)$ are found by dividing this mass by the appropriate density giving

$$
\begin{aligned}
& V_{\mathrm{b}}=\left[\frac{v_{\mathrm{b}} \rho_{\mathrm{b}}}{\left(1-v_{\mathrm{b}}\right) \rho_{\mathrm{i}}+v_{\mathrm{b}} \rho_{\mathrm{b}}}\right]\left[\frac{\rho_{\mathrm{s}}}{\rho_{\mathrm{b}}}\right], \\
& V_{\mathrm{i}}=\left[\frac{\left(1-v_{\mathrm{b}}\right) \rho_{\mathrm{i}}}{\left(1-v_{\mathrm{b}}\right) \rho_{\mathrm{i}}+v_{\mathrm{b}} \rho_{\mathrm{b}}}\right]\left[\frac{\rho_{\mathrm{s}}}{\rho_{\mathrm{i}}}\right] .
\end{aligned}
$$

The volume fraction of air is then simply

$$
V_{\mathrm{a}}=1-\left(V_{\mathrm{b}}+V_{\mathrm{i}}\right) \text {. }
$$

The volume fractions of brine, ice, and air are plotted against snow temperature in Figure 1, for a snow density of $300 \mathrm{~kg} \mathrm{~m}^{-3}$ and salinity of $40 \%$, values found to be typical of the lowest centimetre of the snow-pack.

The most significant feature of the graph with regard to dielectrics is that the volume fraction of brine remains fairly large down to $-22.9^{\circ} \mathrm{C}$, when sodium chloride dihydrate begins to come out of solution. Below this temperature, $V_{\mathrm{b}}$ falls off rapidly but some liquid remains in the snow at temperatures below $-40^{\circ} \mathrm{C}$. It should be noted that the volume fraction of cryohydrates has not been calculated. Removing the appropriate amount of salt from the measured salinity before determining $v_{b}$ has the effect of increasing $\left(1-v_{b}\right)$ and thereby causing a small erroneous increase in $V_{\mathrm{i}}$. When modelling the dielectric properties of the snow-pack, this effect is negligible since it is the liquid fraction which is dominant; however, for other uses it may be necessary to calculate the cryohydrate fraction as well.

The volume fractions of air, ice, and brine for the Resolute Passage data are shown in Figure 2. The consistent increase in density with time in layer 2 indicates the presence of constructive metamorphosis and mass transfers from the base of the snow-pack into the upper layers, a feature confirmed by the observation of a thin depth-hoar layer developing at the snow/ice interface. Although $V_{\mathrm{i}}$ in

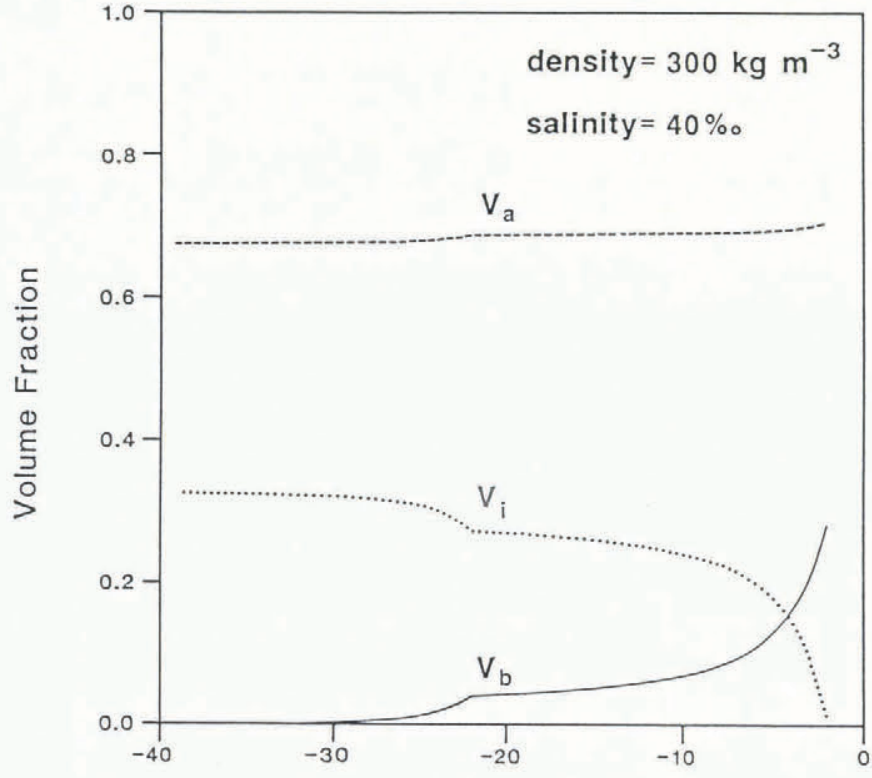

Temperature $\left({ }^{\circ} \mathrm{C}\right)$

Fig. 1. Variation in the volume fractions of brine (b), ice (i), and air (a) with snow temperature, for a constant snow density of $300 \mathrm{~kg} \mathrm{~m}^{-3}$ and salinity $40 \%$.

the bottom layer also increases slightly, this is primarily the result of reduced brine volumes caused by decreasing surface temperatures as the ice thickens. The relatively high brine fractions, particularly in the lowest layer, are clearly evident as are the increases in $V_{\mathrm{b}}$ in all layers between days 24 and 32 . The reasons for this increase are not immediately evident, but may be associated with additional brine being drawn up from the surface by increased capillary tension after the input of new, more angular, crystals of wind-blown snow during a storm which occurred on days 23 and 24 . It is interesting to see that there is a distinct lag in the brine-fraction increase with distance from the ice surface, indicating that the upward flux of brine is slow, taking about a week to reach layer 3 . The gap in the time series in the upper layer represents a period when no snow was present at that level, having been scoured by strong wind.

\section{MODELLING THE DIELECTRIC PROPERTIES OF SALINE SNOW}

The complex dielectric constant $\left(\varepsilon^{*}\right)$ of a medium may be represented simply by $\varepsilon^{*}=\varepsilon^{\prime}-i \varepsilon \varepsilon^{\prime}$, where $\varepsilon^{\prime}$ is the real part of the dielectric constant or permittivity, $\varepsilon$ " is the imaginary part or dielectric loss, and $i=(-1)^{\frac{1}{2}}$. The loss occurring when electromagnetic energy passes through the medium is commonly represented in terms of the loss tangent $(\tan \delta)$, where $\tan \delta=\varepsilon^{\prime \prime} / \varepsilon^{\prime}$.

The snow on young sea ice is a mixture of air, ice, and brine, and its effective complex dielectric constant $\left(\varepsilon^{*}\right)$ is a weighted average of the complex dielectric constants of the constituent parts. Theoretical studies have shown that the dielectric constant of heterogeneous materials is strongly influenced by the way in which the various components are distributed in the mixture, indicating that any rigorous approach to the problem must include both the dielectric properties of the constituent parts and their distribution. This is the approach adopted here, and we begin by looking at the dielectric properties of ice and saline water.

\subsection{Microwave dielectrics of ice and saline water}

Throughout the metre and centimetre wavelength range, the relative complex dielectric constant of ice is invariant (Cumming, 1952; Evans, 1965). The real part of the relative complex dielectric constant $\left(\varepsilon_{\text {ice }}^{\prime}\right)$ is 3.15 , and the dielectric loss is so low that it may be ignored (thus, $\varepsilon_{\text {ice }}^{*}=\varepsilon_{\text {ice }}^{\prime}$ ). The electromagnetic loss tangent $(\tan \delta)$ is typically of the order of $10^{-2}$ to $10^{-4}$, depending on the temperature of the ice, and is several orders of magnitude smaller than the loss 

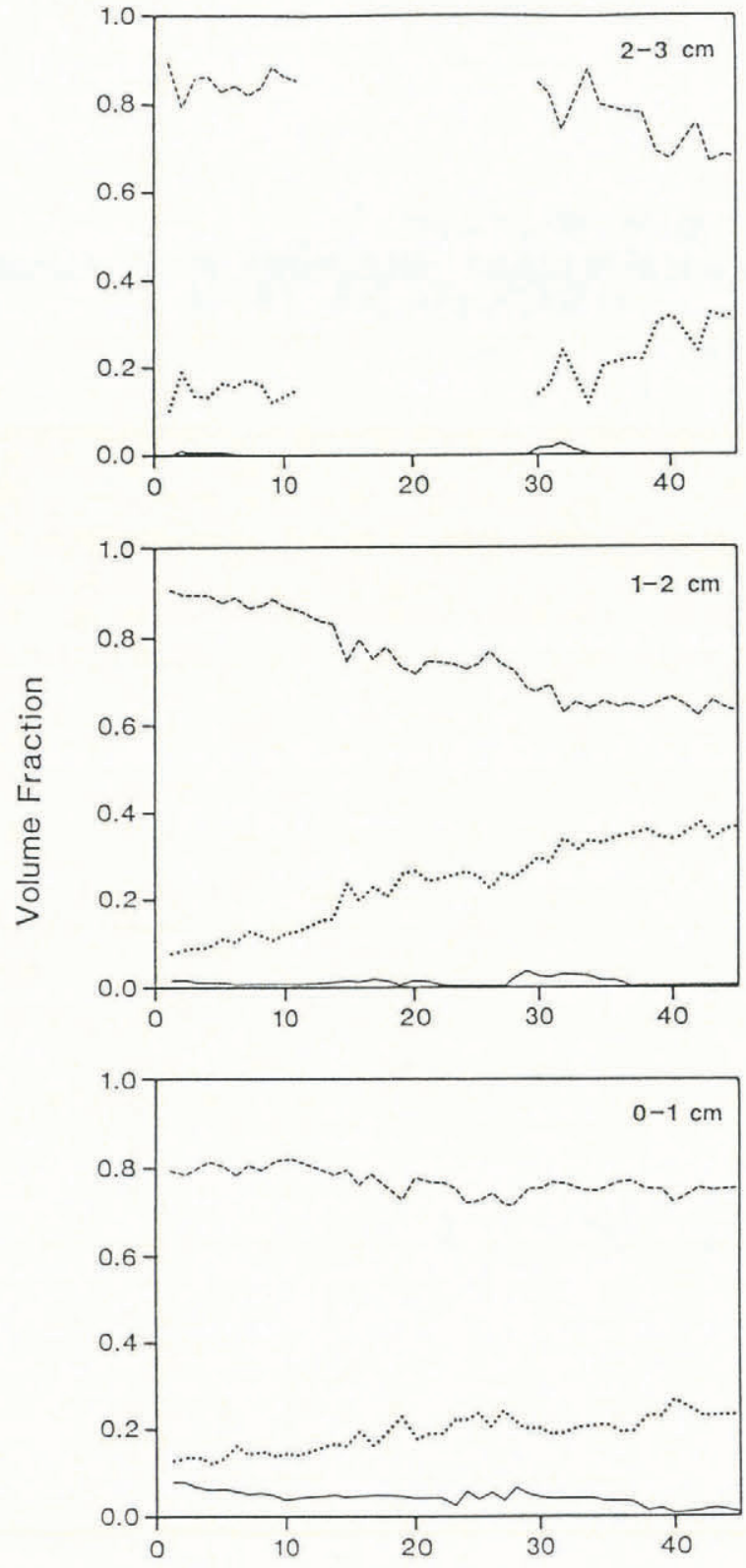

Time (days)

Fig. 2. Variations in the volume fractions of air, ice, and brine for the $46 d$ observation period in layers $0-1.1-2$, and $2-3 \mathrm{~cm}$ measured vertically from the snow/ice interface.

tangent of brine. Tiuri and others (1984) and Hallikainen and others (1982) found that the relative permittivity $\left(\varepsilon_{\mathrm{dry}}^{\prime}\right)$, the real part of the complex dielectric constant, of dry snow mixtures of air and ice can be represented by the simple linear empirical relationship

$$
\varepsilon_{\mathrm{dry}}^{\prime}=1.0+2 p_{\mathrm{dry}}
$$

where $\varepsilon_{d r y}^{\prime}$ is the relative permittivity of dry snow and $\rho_{\text {dry }}$ is its density.

The dielectric behaviour of water as a function of frequency is known in first approximation to conform to a simple Debye relaxation spectrum. The dielectric constant falls off in the dispersion region, whereas the dielectric losses reach a maximum. Salts drastically alter the dielectric loss of water by adding to the free-charge carriers and increasing its conductivity, but the influence on the dielectric constant is small (Hoekstra and Cappillino, 1971).

Equations for computing the complex dielectric constant of saline water have been developed by Stogryn (1971), based on an equation of the Debye form, in which the dielectric properties have a salinity and temperature dependence. The term "saline water" is strictly applicable only to water containing $\mathrm{Na}^{+}$and $\mathrm{Cl}^{-}$ions but, since $\mathrm{NaCl}$ is the primary salt in sea-water, Stogryn's equations for saline water can be used for the brine inclusions in young sea ice and snow without significant error.

To calculate the complex dielectric properties of brine, we have used the following equation (after Stogryn, 1971):

$$
\varepsilon_{b}^{*}=\varepsilon_{\infty}+\frac{\varepsilon_{s}-\varepsilon_{\infty}}{1-i \omega \tau}+i \frac{\sigma}{\omega \varepsilon_{0}}
$$

where $\varepsilon$ is the relative complex dielectric constant of the mixture, and $\varepsilon_{\infty}, \varepsilon_{s}, \varepsilon_{0}, \omega, \tau$, and $\sigma$ are the highfrequency and static relative dielectric constants of the saline water, the permittivity of free space (i.e. $8.85 \times 10^{-12}$ farad $\mathrm{m}^{-1}$ ), the angular frequency, the relaxation time, and the ionic conductivity of the dissolved salts, respectively.

To obtain values for the parameters in Equation (8), Stogryn developed a series of simple polynomial fits to real dielectric data for saline water, with temperature and salinity as input variables. Results from Stogryn's equations at a fixed temperature of $-20^{\circ} \mathrm{C}$ and for salinities of 30 and 100\% are displayed across the $\mathrm{GHz}$ range of frequencies in Figure 3. Vant and others (1978) discussed these equations and their applicability to the problem of calculating the complex dielectric properties of different sea-ice types.

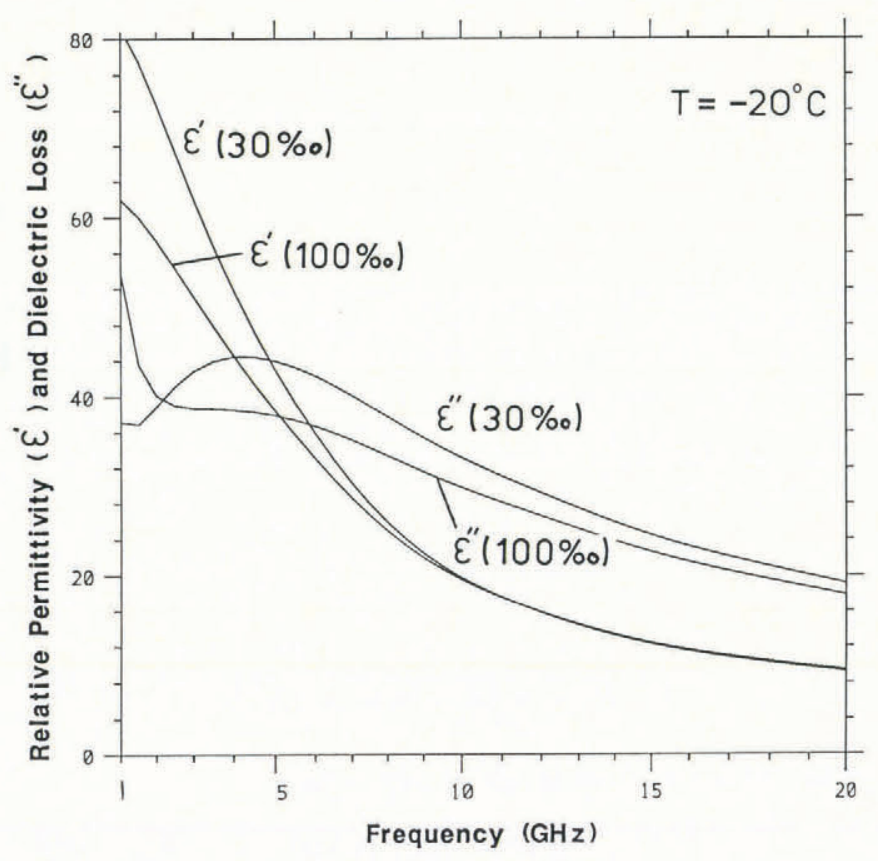

Fig. 3. Behaviour of the relative permittivity ( $\left.\varepsilon_{b}^{\prime}\right)$ and dielectric loss $\left(\varepsilon_{b}^{\prime \prime}\right)$ of brine in the $\mathrm{GHz}$ range: measured at salinities of $30 \%$ and $100 \%$, and a temperature of $-20^{\circ} \mathrm{C}$.

\subsubsection{Application of a mixture formula}

The complex dielectric constant of saline snow (i.e. a mixture of air, ice, and brine) can be determined by a mixing formula which takes into account the specific depolarization due to the liquid inclusions. Interstitial liquid in snow collects around the contact points of the crystals (Drinkwater, 1987), forming complex geometric shapes. Although the shape of brine inclusions in the snow matrix does not conform either to perfect ellipsoids or spheroids, Denoth (1980) suggested that, in the case of low liquid fractions, the arrangement of free water may be assumed to approximate to discs. Therefore, the water menisci will be modelled here as oblate spheroids. Denoth (1980) studied snow of varying water content and derived a formula for calculating the specific depolarization factor of liquid inclusions of known dimensions. He also suggested that, as the liquid fraction in a snow cover increases, both the shape of the liquid inclusions and the depolarization factor change. In this paper, however, we discuss only snow of low density and low liquid-volume fractions, and therefore 
assume that oblate spheroids adequately represent the brine-fraction geometry. A dielectric mixture formula, developed for heterogeneous systems containing dispersed ellipsoids, has been discussed by Van Beek (1967), and a simplified form of this equation, generalized for a uniform distribution of orientations of liquid inclusions in space, was utilized with success by Chaloupka and others (1980), and more recently by Mätzler and others (1984), for wet snow. Polder and van Santen (1946) showed that this type of formulation holds true for low volume fractions of water, and may also accommodate shapes of water inclusions such as those described by Denoth (1980). The formula used to calculate the complex dielectric constant of the mixture $\left(\varepsilon_{\text {mix }}^{*}\right)$ is

$$
\varepsilon_{\text {mix }}^{*}=X V_{\mathrm{b}}\left[\frac{\varepsilon_{\mathrm{b}}^{*}-\varepsilon_{\mathrm{dry}}^{\prime}}{1+\left(\frac{\varepsilon_{\mathrm{b}}^{*}}{\varepsilon_{\mathrm{dry}}^{\prime}}-1\right) A_{0}}\right]
$$

where $A_{0}$ is the dominant depolarization factor, $V_{\mathrm{b}}$ is the volume fraction of brine, $\varepsilon_{b}^{*}$ is the complex dielectric constant of brine, $\varepsilon_{\text {dry }}^{\prime}$ is the relative permittivity of dry snow (from Equation (7)), and $X$ is a coupling factor representing the fraction of brine that can be represented by $A_{0}$. For isotropically oriented oblate spheroids, the coupling factor $(X)$ is $2 / 3$. In the case of snow in the pendular regime, Denoth observed a depolarization factor $A_{0}$ of approximately 0.053 .

The temporal variations in the dielectric properties of the snow cover on young sea ice are modelled using Equation (9), along with the calculated volume fractions of air, ice, and brine from the Resolute Passage data.

\subsection{Discussion of results from mixture formula}

As temperature, salinity, brine volume, and density vary over the $45 \mathrm{~d}$ period (Fig. 2), we see marked changes in the modelled complex dielectrics of the surface snow medium. This is shown in Figures 4 and 5 , at $1 \mathrm{GHz}$ (L-band) and $13.6 \mathrm{GHz}$ (Ku-band), respectively, where the gradual evolution of the layer is reflected in the changing electromagnetic properties.

The changes in the imaginary part $(\varepsilon$ ") of the relative complex dielectric constant, albeit small, are indicative of the amount of brine in the snow. Although the dielectric loss is slightly larger at higher frequencies, owing to the relaxation mechanism in brine, there is a gradual reduction in $\varepsilon$ " with time over all frequencies.

In direct contrast, the dielectric constant $\left(\varepsilon^{\prime}\right)$ is larger
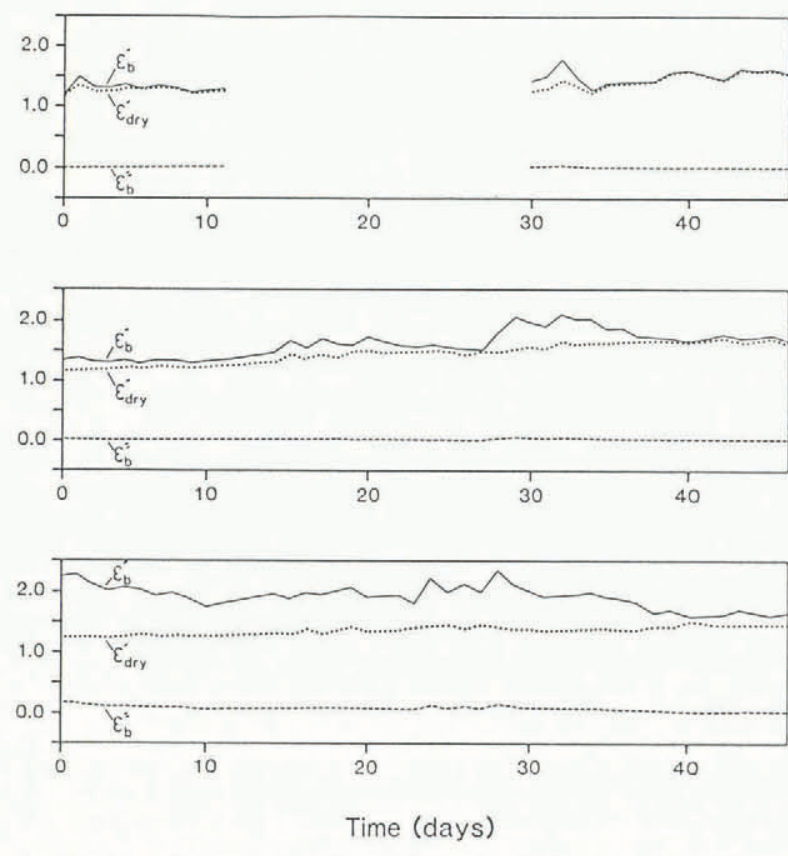

Fig. 4. Predicted variations in $\varepsilon_{b}^{\prime}$, $\varepsilon_{\mathrm{dry}}^{\prime}$, and $\varepsilon_{b}^{\prime \prime}$ over the $46 d$ period. Values are calculated for the snow layers $0-1,1-2$, and $2-3 \mathrm{~cm}$ at a frequency of $1 \mathrm{GHz}$.
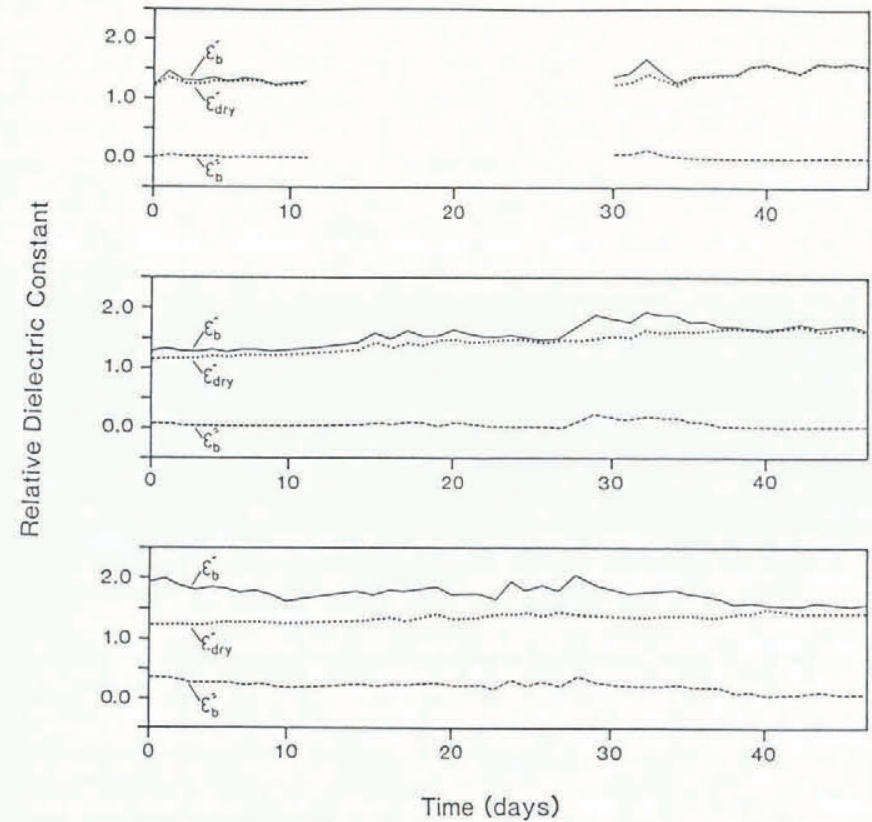

Fig. 5. Predicted variations in $\varepsilon_{b}^{\prime}, \varepsilon_{\mathrm{dry}}^{\prime}$, and $\varepsilon_{b}^{\prime \prime}$ over the $46 d$ period. Values are calculated for the snow layers $0-1,1-2 \mathrm{~cm}$, and $2-3 \mathrm{~cm}$ at a frequency of $13 \mathrm{GHz}$.

at lower frequencies due to large increases in the dielectric constant of brine below $10 \mathrm{GHz}$ (X-band). Generally, $\varepsilon^{\prime}$ increases in the upper $2 \mathrm{~cm}$, tending to the values for dry snow by the end of the observation period. This is due to the continual densification of the snow layer and the decline in brine volumes. The dielectric constant in the layer directly above the saline surface of the frazil, however, shows a small decrease; in this case, the reduction in brine volume dominates over the slight change in density and causes $\varepsilon^{\prime}$ to fall by around 0.5 .

In the early part of the measurement period, the difference between the dielectric constants of brine-wetted snow and dry snow will result in increased reflectivity at the air/snow interface. Despite the fact that the mean transmissivity of the snow is generally high $(\approx 0.8-0.9)$, the layer will effectively blanket emission or surface scattering from the saline frazil-ice medium beneath as a result of the high extinction coefficient and lossy nature of saline snow. As time progresses and $V_{\mathrm{b}}$ decreases, the snow approaches values observed in higher-density dry snow $\left(\varepsilon^{\prime} \simeq 1.6\right.$ and $\varepsilon^{\prime \prime} \simeq 0.01$ )

It is clear that the complex dielectric constant of the mixture is largely dependent upon the brine volume and to a lesser extent upon the density of the snow. As brine volume decreases over the observation period, the dielectric properties of the snow cover tend to those of dry snow of similar densities. At lower frequencies, the differences between the relative dielectric constants of brine-wetted snow and dry snow are exacerbated because of the higher dielectric constant of brine.

\section{SCATTERING FROM YOUNG ICE SURFACES}

The radar back-scatter properties of cold, thin firstyear ice have been investigated experimentally by Onstott and others (1982), and in the $8-18 \mathrm{GHz}$ range by Delker and others $(1980 \mathrm{a}, \mathrm{b})$, off the Canadian coast at Tuktoyaktuk, N.W.T. The site chosen was a frozen lead, covered with frost flowers of high salinity, a similar situation to that observed in Resolute Passage. Ice-surface temperatures ranged between $-20^{\circ}$ and $-30^{\circ} \mathrm{C}$, but a detailed description of the ice or snow densities, surface-roughness properties, snow depth, or snow wetness were unfortunately not given. They observed a strong correlation between radar cross-sections of sea ice and the salinity of the ice surface. In general, higher-salinity thin ice produced higher scattering coefficients than older thicker first-year ice, although the trends of back-scatter coefficient with incidence angle demonstrated parallel responses, i.e. equal angular decays. 
Surface roughness is understood to have an important influence on radar scattering from saline-ice surfaces (Kim and others, 1985), or wet snow surfaces (Stiles and Ulaby, 1980). The appearance of any given surface to incident microwave energy may be evaluated using the Rayleigh criterion:

$$
\sigma>\frac{\lambda}{8 \cos \theta} \text { (for the surface to appear rough) }
$$

where $\sigma$ is the standard deviation of surface heights and $\theta$ is the incidence angle measured from the normal. The parameters used to describe the snow and ice surfaces are: the standard deviation of small-scale surface height and slope, and the spatial autocorrelation function of the surface, from which the correlation length $(l)$ can be derived. Approximate values derived from close-up photography of early frost-flowered surfaces at the sample site were $\sigma \simeq 0.5 \mathrm{~cm}$ and $l \simeq 5.0 \mathrm{~cm}$. For the frequencies discussed in this study, the snow surface may be considered "rough" for $\theta<56^{\circ}$ at $\mathrm{Ku}$-band $(\lambda \approx 2.2 \mathrm{~cm}), \quad \theta<36^{\circ}$ at X-band $(\lambda \approx 3.2 \mathrm{~cm})$, and effectively smooth for all angles at C-band $(\lambda \approx 5.6 \mathrm{~cm})$ and L-band $(\lambda \approx 30 \mathrm{~cm})$. Strictly speaking, the separation between specular and diffuse surfaces is not so rigidly defined, and back-scattered energy is almost always distributed over the full range of incidence angles, with a maximum at normal incidence. In addition, the deposition of wind-blown snow changes surfaceroughness characteristics throughout the observation period, making characterization difficult without a series of measurements of $\sigma$ and $l$.

$\mathrm{Kim}$ (unpublished) asserted that surface scattering is liable to be the major back-scattering mechanism for snowfree first-year ice because of the dielectric mismatch between air and a high-salinity ice medium such as frazil ice. The large dielectric constant at the surface of saline ice leads to high reflection coefficients, and thus low transmissivities or emissivities. At a typical density of $850 \mathrm{~kg} \mathrm{~m}^{-3}$ and salinity of $4 \%$, the reflection coefficient of frazil ice at normal incidence tends to a minimum of approximately $-11.5 \mathrm{~dB}$ at temperatures below $-40^{\circ} \mathrm{C}$, and a maximum of almost $0 \mathrm{~dB}$ at $0^{\circ} \mathrm{C}$. Several attempts have been made to explain radar back-scatter behaviour for saline ice (Fung and Eom, 1982; Kim and others, 1984, 1985; Parashar, unpublished), all based upon theoretical models of scattering and incorporating work on the physical and electromagnetic properties of ice. Kim (unpublished) demonstrated that physical optics formulations, as reproduced by Ulaby and others (1982), can be used to give first-order predictions of the microwave back-scatter of first-year ice. In this paper, we use a similar Kirchoff-type formulation and a "scalar approximation" (Eom, unpublished). The criteria which must be satisfied for the use of such a theoretical model are that the radius of curvature at all points upon the surface are larger than one wavelength, and that the standard deviation of surface heights is small, or of comparable size to the wavelength (Beckmann and Spizzichino, 1963; Ulaby and others, 1982). Ulaby and others (1982) also suggested that, for any general Kirchoff-type formulation to be used, $k_{0} l$ must be greater than or equal to 6 , where $k_{0}$ is the wave number in air (i.e. $2 \pi / \lambda$ ) and $l^{2}>2.76 \sigma \lambda$. More specifically, the r.m.s. surface slope must not exceed 0.25 for a scalar approximation to be implemented. For the range of surface characteristics observed at Resolute Passage, these criteria are met at $\mathrm{Ku}-, \mathrm{X}$-, and $\mathrm{C}$-band, although not at $\mathrm{L}$-band. This suggests that we are justified in using this approximation in the model to indicate the magnitude of scattering from these surfaces.

Young ice and nilas thicker than a few centimetres might be expected to demonstrate similar emissivity and back-scattering properties. Ramseier and others (1975) and Ketchum and Lohanick (1980), however, have observed emissivities for young ice which differ significantly from older first-year ice. They attributed these differences to the presence of moisture on the surface of the young ice, which lowers its emissivity. This suggests that the brine content observed in the snow on Resolute Passage may cause significant changes in the emissivity of the mixture, and that the effects of a highly saline snow layer on the microwave signatures of young ice surfaces must be examined more closely. Also, Kim and others (1984) and
Campbell and others (1978) have indicated that a dry snow cover has a significant effect on the microwave signature of an ice surface. In the case of active instruments, the effect may increase the back-scatter coefficient by several dB. However, little data are available on the effects of a saline snow cover upon back-scattering mechanisms from young sea-ice forms. In the next section, we model the scattering properties of saline, snow-covered young ice forms, and trace the likely changes in back-scatter signatures as the snow layer upon it evolves. A parametric study is conducted to suggest the possible range of the scattering coefficient $\sigma^{\circ}$ over the $46 \mathrm{~d}$ period.

\subsection{Snow-covered sea-ice back-scattering model}

Scattering from snow-covered sea ice has three sources: surface scatter from the air/snow interface, volume scatter by inhomogeneities within the snow layer, and surface scatter from the snow/ice interface (providing the losses in the snow layer are small). Scattering by the uppermost interface is governed by the dielectric mismatch and the surface geometry (Ulaby and others, 1984). Despite varying salinities, the dielectric mismatch between air and snow is still relatively small even at day 1 when $\varepsilon_{\text {air }}^{\prime}=1$ and $\varepsilon_{\text {snow }}^{\prime} \simeq 2.0$ (Fig. 4). The snow-surface back-scattering contribution is therefore likely to be concentrated at nearnormal incidence.

Application of theoretical models to the back-scattering behaviour of snow-covered young ice is difficult, since a detailed data set on the statistical properties of the upper surfaces of "the snow and sea-ice layers, and of the particle-size distribution of the ice crystals, are necessary. Therefore, by modelling the back-scatter response, we intend merely to indicate first-order effects, rather than accurate absolute values. General models ignore diffuse scattering in the snow volume, and multiple scattering and reflection in the region between the air/snow and snow/ice interfaces. The back-scatter coefficient is composed of three terms: (i) a surface component from snow/air interface $\left(\sigma_{\mathrm{sa}}^{\circ}\right)$, (ii) a volume-scatter component from within the snow layer $\left(\sigma_{\mathrm{s}}\right)$, and (iii) a scatter component from the distinct underlying frazil-ice surface $\left(\sigma_{i}^{\circ}\right)$. Ulaby and others (1984) and Drinkwater (unpublished) expressed the total coefficient of back-scatter $\left(\sigma_{\text {tot }}^{\circ}\right)$ as:

$$
\sigma_{\mathrm{tot}}^{\circ}(\theta)=\sigma_{\mathrm{sa}}^{\circ}(\theta)+\sigma_{\mathrm{S}}^{\circ}\left(\theta^{\prime}\right)+\frac{\mathrm{Y}_{\mathrm{sa}}^{2}\left(\theta^{\prime}\right)}{L^{2}\left(\theta^{\prime}\right)} \sigma_{\mathrm{i}}^{\circ}\left(\theta^{\prime}\right)
$$

where $Y_{s a}^{2}\left(\theta^{\prime}\right)$ is the power-transmission coefficient at the snow-air boundary, $L\left(\theta^{\prime}\right)$ is the one-way loss factor of the snow layer, and $\theta^{\prime}$ is the complex transmission angle in the snow layer (which is related to the angle of incidence $\theta$ through Fresnel formulae). Furthermore, $\sigma_{\mathrm{S}}^{\circ}\left(\theta^{\prime}\right)$ and $L\left(\theta^{\prime}\right)$ are:

$$
\sigma_{\mathrm{S}}^{\circ}\left(\theta^{\prime}\right)=\mathrm{Y}_{\mathrm{sa}}^{2}\left(\theta^{\prime}\right)\left[\frac{\sigma_{\mathrm{v}}}{2 \mathrm{k}_{\mathrm{e}}}\left(1-\frac{1}{L^{2}\left(\theta^{\prime}\right)}\right) \cos \theta^{\prime}\right],
$$

$$
L\left(\theta^{\prime}\right)=\exp \left(\boldsymbol{k} \mathrm{e}^{d \sec \theta^{\prime}}\right)
$$

where $\sigma_{\mathrm{v}}$ is the volume-scatter coefficient or radar reflectivity of the snow layer, $k_{e}$ is the extinction coefficient, and $d$ is the depth of the snow layer. The value of $\sigma_{\mathrm{v}}$ is found using equations similar to those of Ulaby and others (1982) and Kim and others (1985), based on the radiative-transfer model (Karam and Fung, 1982). Ignoring multiple scattering and assuming that the individual scatterers are spherical equidimensional, and distributed uniformly throughout the layer (Attema and Ulaby, 1978), $\sigma_{\mathrm{v}}$ is given by:

$$
\sigma_{\mathrm{v}}=N_{1} \sigma_{\mathrm{b}_{1}}+N_{2} \sigma_{\mathrm{b}_{2}}
$$

where $N$ is the number of scatterers per unit volume, and $\sigma_{b}$ is the back-scattering cross-section of a single particle, and the subscripts 1 and 2 denote ice crystals and brine inclusions, respectively. Scattering contributions are made by 
both ice particles and brine inclusions at the snow-grain boundaries, and are combined to give a total volume-scatter component. Ulaby and others (1982) suggested a "particle cloud" analogy of this nature is particularly appropriate for snow volumes, since they consist in reality of scattering particles - the ice crystals - in an air background. We include an additional term for the effects of the brine inclusions as independent scatterers. The relative merits and shortcomings of this approach to modelling snow-volume scatter, or its overall applicability in the context of snow covers on sea ice, will not be discussed here. We merely wish to demonstrate the relative back-scatter contribution of a brine-wetted snow cover upon young ice forms. This approximation is particularly suitable because of its simplicity and, because the size of the scatterer is small relative to a wavelength, we are able to calculate $\sigma_{b}$ and $\sigma_{\mathrm{b}_{2}}$ from Rayleigh approximations (Van de Hulst, 1957). ${ }^{1}$
The surface-scattering terms in Equation (11) were calculated using the physical optics model (Ulaby and others, 1982). Since no detailed temporal statistics are available for cold saline snow surfaces, we shall assume a Gaussian distributed randomly rough surface with correlation length $l$, height elements $h$, and height distribution

$$
p(h)=\left(2 \pi \sigma^{2}\right)^{-1 / 2} \exp \left(-h^{2} / 2 \sigma^{2}\right)
$$

where $\sigma^{2}$ is the variance of surface heights. The combined equations used for non-coherent scattering may be found in Ulaby and others (1982), and so are not reproduced here. Other parameters used to generate model back-scatter signatures include the surface-roughness statistics, the mixture and ice dielectrics, and snow- and ice-density values. The ice surface itself maintained consistent values of salinity and density, and we use static values of $10 \%$ and
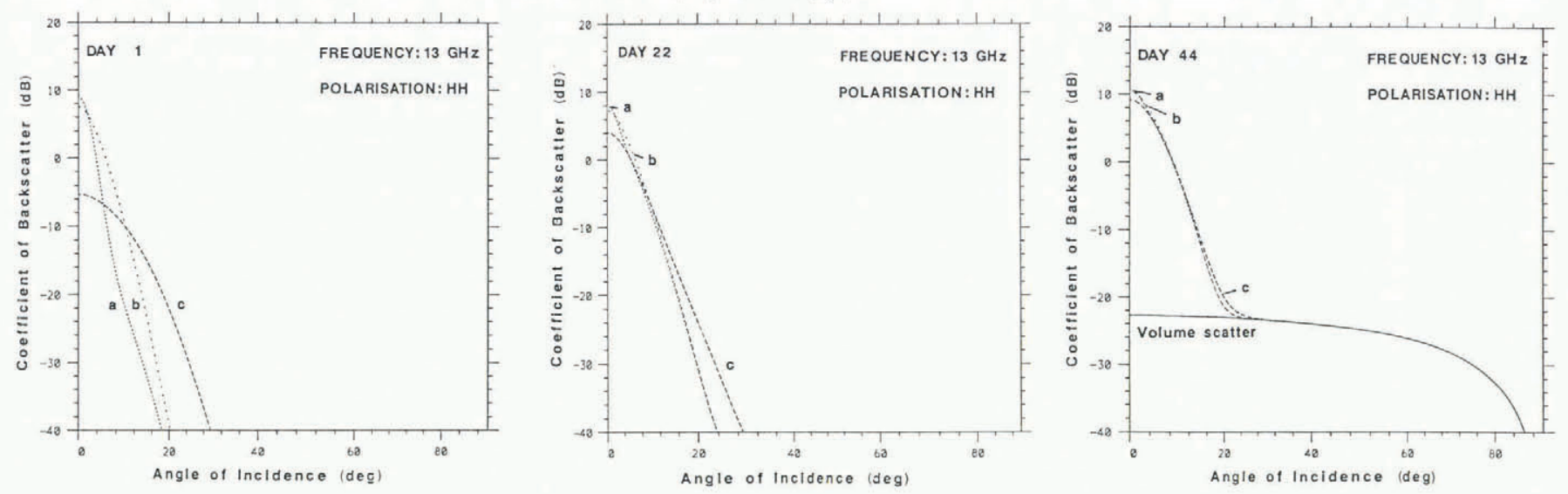

Fig. 6. Theoretical behaviour of $\sigma^{\circ}$ with incidence angle at $13 \mathrm{GHz}$ and $\mathrm{HH}$ polarizalion. The ice surface has constant values of $\sigma=0.37 \mathrm{~cm}$ and $l=8.5 \mathrm{~cm}$, and the three plots $a, b$, and $c$ represent the back-scatter responses for smooth $(\sigma=0.15 \mathrm{~cm}, l=8.5 \mathrm{~cm})$, medium rough $(\sigma=0.37 \mathrm{~cm}$. $l=8.5 \mathrm{~cm})$, and rough $(\sigma=0.81 \mathrm{~cm}, l=8.2 \mathrm{~cm})$ snow surfaces, respectively.
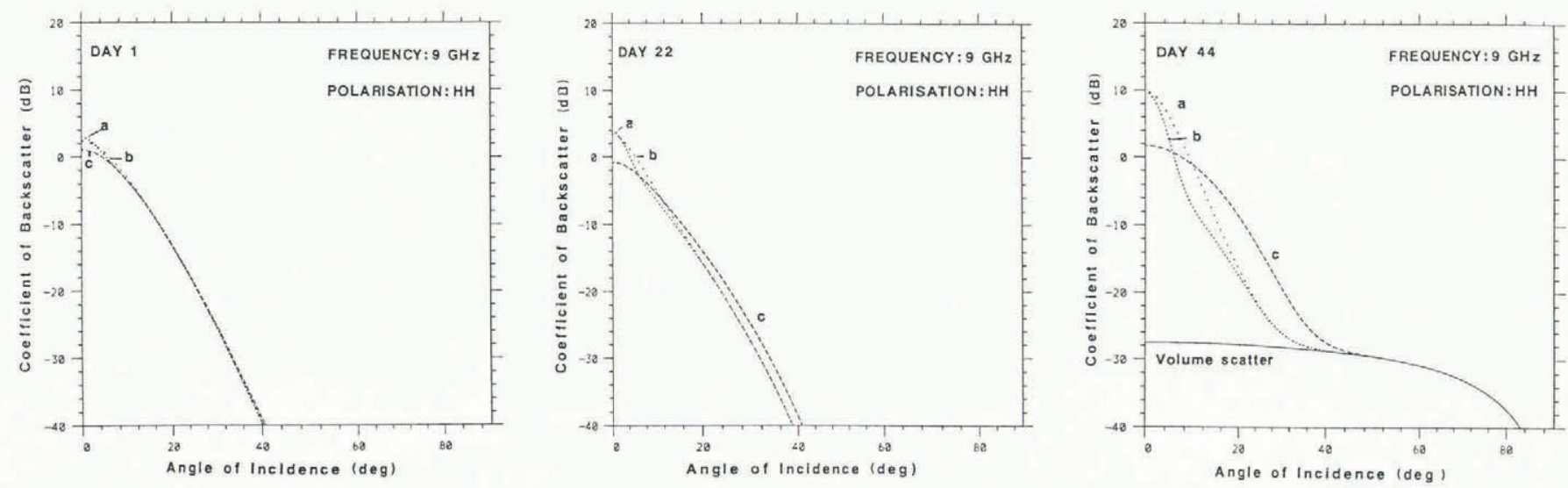

Fig. 7. Theoretical behaviour of $\sigma^{\circ}$ with incidence angle at $9 \mathrm{GHz}$ and $\mathrm{HH}$ polarization for a snow surface of roughness $\sigma=0.5 \mathrm{~cm}$ and $l=5.0 \mathrm{~cm}$. Plots for day 1 , day 22, and day 44 indicate the response to three grades of ice-surface roughness corresponding to the rough, medium rough, and rough surfaces described in Figure 6. 
$910 \mathrm{~kg} \mathrm{~m}^{-3}$ throughout the observation period. From these we are able to calculate the dielectrics of the ice medium (from Vant and others, 1978), which are not found to vary significantly over the GHz-frequency range. Three scales of surface roughness are used as model inputs. Although derived from summer ice surfaces (Kim, unpublished) they are useful in demonstrating the range of values observed under natural circumstances, and as such assist this parametric exercise. The "smooth" surface we consider has values of $\sigma=0.15 \mathrm{~cm}$ and $l=8.9 \mathrm{~cm}$, while the "medium rough" and "rough" surfaces have values of $\sigma=0.37 \mathrm{~cm}$ and $l=8.5 \mathrm{~cm}$, and $\sigma=0.81 \mathrm{~cm}$ and $l=8.2 \mathrm{~cm}$, respectively.

\subsection{Model indications}

Figures 6 and 7 display the predicted behaviour of $\sigma^{\circ}$ with incidence angle, each at three different stages in the period of observations, and at 13 and $9 \mathrm{GHz}$, respectively. Figure 6 shows that snow-surface roughness changes influence surface scattering only during the early stages of snow-cover development. After $22 \mathrm{~d}$, the effect of varying snow-surface statistics has little effect on the modelled back-scattering. This phenomenon can be explained by the fact that despite high transmissivities at the surface, the penetration depth is minimal owing to the brine content of the snow cover. Back-scatter is high at angles close to normal incidence, and back-scatter fall-off with angle is rapid. Thus, despite increasing dry-snow permittivities due to densification, it appears that the angular back-scatter signature is controlled primarily by the brine content. As the snow cover evolves, the brine content decreases and, despite increases in snow density, the contribution from snow-surface scatter is reduced. At this later stage of snowcover development, the effects of surface roughness become negligible, as the reduced brine-volume fractions allow incident energy to penetrate as far as the frazil-ice surface beneath.

A very different situation can be seen in Figure 7 . Because of extinction and absorption in the snow layer during the first $10 \mathrm{~d}$ (when wicking of brine strongly influences permittivities), the ice-surface roughness has a negligible effect on the angular variation in the backscatter coefficient. However, beyond day 10, our analyses suggest that the effects of brine volume are sufficiently reduced for small variations in the scatter signatures to be observed. Contributions from ice-surface scatter are, nonetheless, only observed between $0^{\circ}$ and $20^{\circ}$. For incidence angles greater than about $30^{\circ}$, we calculate that the effects of surface scattering from beneath such a snow layer of $0.03 \mathrm{~m}$ or more in depth are negligible. This applies to ice-surface roughness normally encountered on young ice, since most of the energy directed towards the interface at the transmission angle is reflected away from the instrument.
The upper snow-surface scattering term dominates in the early period $(1-10 \mathrm{~d})$, because of the high brine-volume fractions and increased extinction in the snow layer. The angular response of $\sigma^{\circ}$ is very narrow and typically falls by $50 \mathrm{~dB}$ in the first $20^{\circ}$ away from the normal. For rougher surfaces $\sigma^{\circ}$ does, however, demonstrate lower gradients, but at typical synthetic aperture radar (SAR) or side-looking airborne radar (SLAR) incidence angles of between $30^{\circ}$ and $70^{\circ}, \sigma^{\circ}$ is negligible at $\mathrm{C}-, \mathrm{X}-$, and $\mathrm{Ku}$-bands for the first $20 \mathrm{~d}$.

It is not until after $25 \mathrm{~d}$ have passed that the volumetric scatter contribution from the snow layer becomes significant (see Figs 6 and 7). The continuing densification and concurrent brine-volume reduction up to day 46 lead to higher volume back-scatter values. Extinction coefficients are minimized and scattering by the snow volume contributes to maximum observed values for $\sigma_{\mathrm{v}}^{\circ}$ of around $-20 \mathrm{~dB}$ by day 46 .

The three-dimensional trend surfaces in Figures 8 and 9 describe the predicted transitions occurring in the backscatter signatures over the $46 \mathrm{~d}$ period, for $\mathrm{Ku}-$ and C-band (for $\mathrm{HH}$ polarization). For $13 \mathrm{GHz}$ (Fig. 8), the overall trend is toward a small increase in $\sigma^{\circ}$ at normal incidence, and a fairly stable angular fall-off between $10^{\circ}$ and $30^{\circ}$, which is composed entirely of surface scatter from the two dielectric interfaces. In contrast, $\sigma^{\circ}$ is seen to increase almost linearly with time in the angular interval $35-90^{\circ}$. Small daily events observed in the original sampled data are also seen to contribute to day-to-day fluctuations, manifested as small undulations in the three-dimensional plot.

In the lower-frequency $\mathrm{X}$ - and $\mathrm{C}$-bands, reductions in the magnitude of both the small-scale fluctuations and the long-term increases over $46 \mathrm{~d}$ can be seen. At X-band, the volume-scattering contribution does not feature as prominently as at higher frequencies, attaining maximum predicted values of the order of $10 \mathrm{~dB}$ lower than at $\mathrm{Ku}$-band by the end of the $46 \mathrm{~d}$ period. Increasing the wavelength to $5.66 \mathrm{~cm}$ (C-band) leads to the situation where the contrast in scattering conditions over the total observation period is negligible. Figure 9 illustrates this trend with $\sigma^{\circ}$ displayed as almost the same linear function of $\theta$ throughout the $46 \mathrm{~d}$. Importantly, the volume-scattering contribution remains below $-40 \mathrm{~dB}$, since the scattering efficiency of the snow crystals is reduced the longer the wavelength.

Finally, we highlight the respective contributions of both surface and volume scattering for snow surfaces of differing degrees of roughness, at selected stages of the $46 \mathrm{~d}$ period. Figure 10 illustrates that a reversal occurs in this $3 \mathrm{~cm}$ brine-wetted snow layer, whereupon volume scatter increases above surface scatter. This reversal shifts in frequency as the physical and dielectric properties of the snow layer evolve.
$13 \mathrm{GHz}$

HH Polarisation

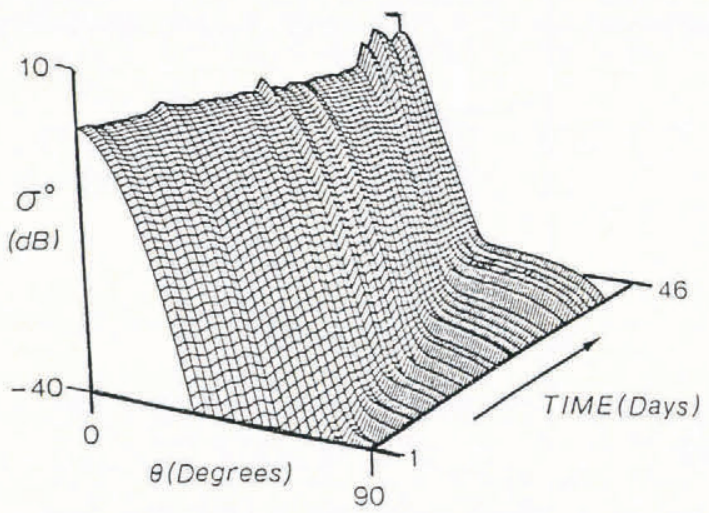

Fig. 8. Trend surface in three-dimensional space illustrating changes of $\sigma^{\circ}$ with time over the range $0-90^{\circ}$ of incidence angles at a frequency of $13 \mathrm{GHz}$.
$5 \cdot 3 \mathrm{GHz}$

HH Polarisation

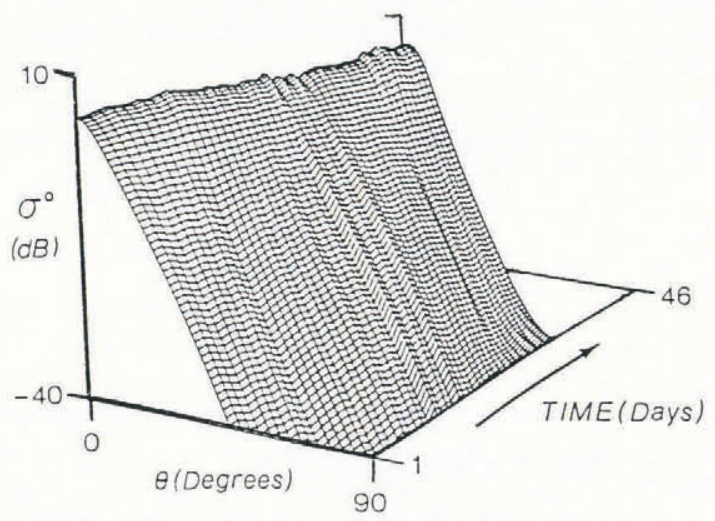

Fig. 9. Trend surface in three-dimensional space illustrating changes of $\sigma^{\circ}$ with time over the range $0-90^{\circ}$ of incidence angles at a frequency of $5.3 \mathrm{GHz}$. 

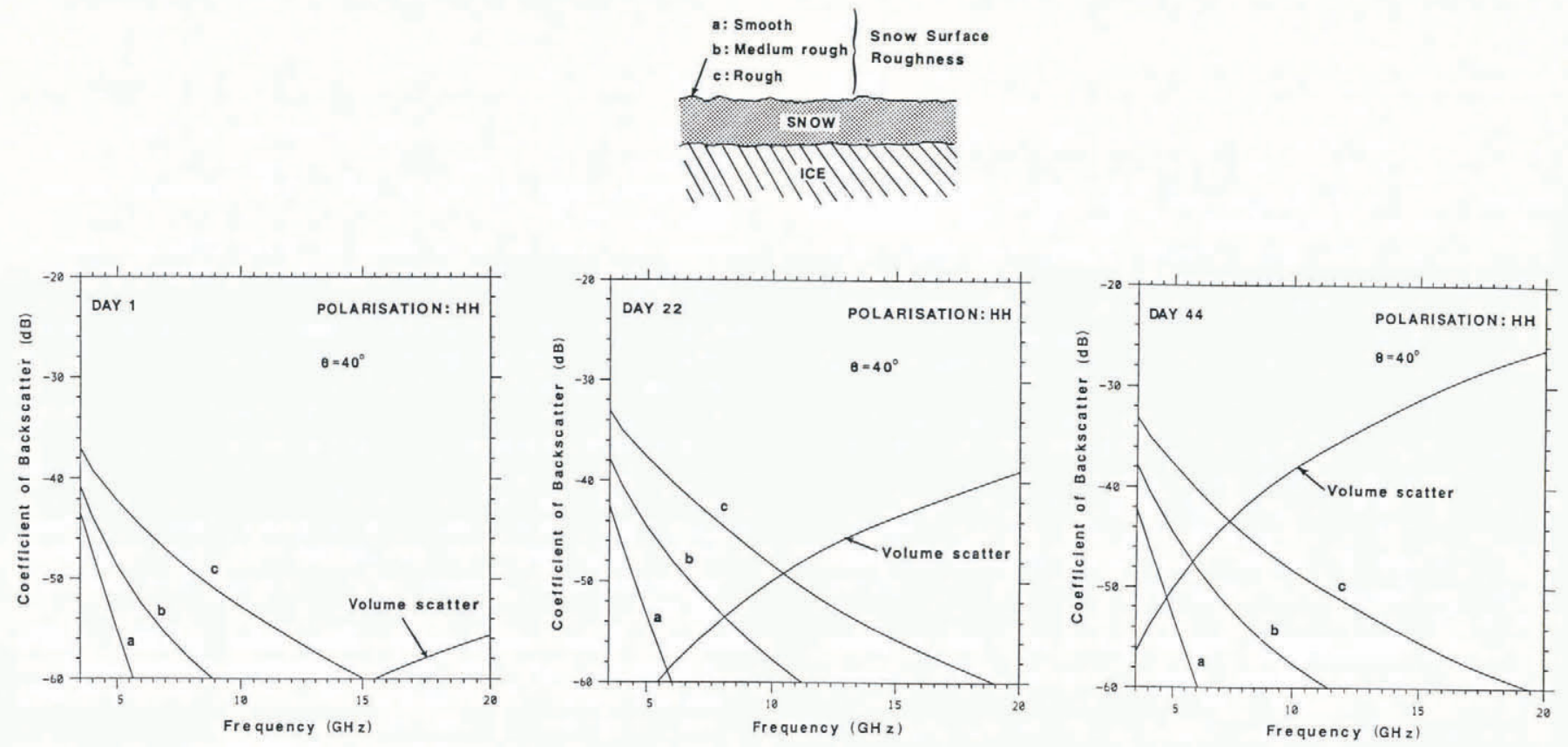

Fig. 10. Relative contributions of surface and volume scatter for the evolving snow layter for days 1 .

22 , and 44 over the $\mathrm{GHz}$ frequency range. Snow-surface roughness varies while ice-surface roughness remains constant at $\sigma=0.37 \mathrm{~cm}, l=8.5 \mathrm{~cm}$.

\section{CONCLUSIONS}

We have observed a number of important changes in the physical and electromagnetic properties of a snow cover on cold, young sea ice which lead to significant variations in the surface-scattering properties. The modelled backscatter and dielectric properties are first approximations based on the conditions found in Resolute Passage during the winter of 1982, and cannot be assumed to be representative of all young, snow-covered sea ice. Although the results of Delker and others $(1980 \mathrm{a}, \mathrm{b})$ are available for comparison, the ice temperature and salinity conditions at their site, which are critical to scattering, were not exactly the same. We cannot, therefore, draw direct comparisons between our results and their measured back-scatter values.

Small-scale roughness plays an important role in determining the back-scatter coefficient during the early stages of ice and snow-cover development in Resolute Passage, and detailed statistics must evidently be obtained for the roughness of snow-covered young ice forms before more accurate absolute values of $\sigma^{\circ}$ can be predicted. Recently, Onstott and others (1983) measured standard deviations of surface heights and surface-correlation lengths on snow-free summer and fall ice surfaces, and found that calculated correlation functions displayed exponential decay. However, since no statistics are available for cold snow-covered thin ice surfaces, there is no a priori justification for using a Gaussian or exponential correlation function in the scattering model at this stage. As even with an exponential correlation function, surface roughness may account for as much as $15 \mathrm{~dB}$ difference in $\sigma^{\circ}$ at large incidence angles (Kim, unpublished), this is an important problem and one that will only be resolved with the collection of more detailed surface-roughness information. Our models suggest that small variations may also be caused by brine-volume, temperature, and density variations, but not the bulk properties of the ice, which remained nearly constant during the study period.

These findings have a number of implications for the interpretation of SAR and SLAR imagery, particularly where it is necessary to distinguish young ice forms from either open water or thicker first-year ice. The results show that further work on the seasonal variations in the physical and dielectric properties of snow-covered sea ice and their effects on the back-scatter from the surface is required before ice types can be identified, without ambiguity, from radar imagery.

\section{ACKNOWLEDGEMENTS}

M.R.D. acknowledges support from a U.K. Natural Environment Research Council studentship. The field part of the project was funded by the Arctic Institute of North America, the Department of Indian and Northern Affairs (Canada), and the Natural Sciences and Engineering Research Council of Canada. Logistical support was provided by the Polar Continental Shelf Project, and field assistance was provided by A.T. Roberts. We also wish to thank F. Carsey, J.G. Paren, and J. Bamber for helpful discussions and suggestions during this work.

\section{REFERENCES}

Arctowski, H. 1908. Les glaces. Glace der mer et banquises. In Résultats du voyage du S.Y. Belgica en 1897-1898-1899. Rapports Scientifiques. [Vol. 5.]

Assur, A. 1960. Composition of sea ice and its tensile strength. CRREL Res. Rep. 44.

Attema, E.P.W. and F.T. Ulaby, 1978. Vegetation modelled as a water cloud. Radio Sci., 13, 357-364.

Beckmann, P. and A. Spizzichino. 1963. The scattering of electromagnetic waves from a rough surface. London, Pergamon Press.

Beek, L.K.H. van. 1967. Dielectric behaviour of heterogeneous systems. In Birks, J.B., ed. Progress in dielectrics. Vol. 7. London, Heywood Books, 69-114.

Campbell, W.J., and 19 others. 1978. Microwave remote sensing of sea ice in the AIDJEX Main Experiment. Boundary-Layer Meteorol., 13(1-4), 309-337.

Chaloupka, H., O. Ostwald, and B. Schiek. 1980. Structure independent moisture measurements. J. Microwave Power, 15, 221-231.

Collinson, R. 1889. Journal of the HMS Enterprise ... 18501855. London, Sampson Low, Marston, Searle and Rivington.

Crocker, G.B. 1984. A physical model for predicting the thermal conductivity of brine-wetted snow. Cold Reg. Sci. Technol., 10(1), 69-74.

Crocker, G.B. Unpublished. The physical properties of snowcover on sea ice in the central high Arctic. (M.Sc. thesis, McGill University, 1984.)

Crocker, G.B. and J.E. Lewis. 1985. Some physical properties of snowcover on evolving first-year sea ice, Proc. East. Snow Conf., 42nd annual meeting, 84-95. 
Cumming, W.A. 1952. The dielectric properties of ice and snow at 3.2 centimetres. J. Appl. Phys., 23(7), 768-773.

Delker, C.V., R.G. Onstott, and R.K. Moore. 1980a. Intermediate results on the radar backscatter study of the sea ice in the Beaufort Sea. Univ. Kans. Remote Sensing Lab. Tech. Rep. 331-15.

Delker, C.V., R.G. Onstott, and R.K. Moore. 1980b. Radar scatterometer measurements of sea ice: The SURSAT Experiment. Univ. Kans. Remote Sensing Lab. Tech. Rep. 331-17.

De Long, G.W. 1883. The voyage of the Jeanette. Vol. 1 . London, Kegan Paul, Trench and Company.

Denoth, A. 1980. The pendular-funicular liquid transition in snow. J. Glaciol., 25(91), 93-97.

Drinkwater, M.R. 1987. Observations of floe surface snow characteristics. In Holt, B., ed. LIMEX '87 Data Report; RADARSAT Project Office Report.

Drinkwater, M.R. Unpublished. Radar altimetric studies of polar ice. (Ph.D. thesis, University of Cambridge, 1988.)

Eide, L.I. and S. Martin. 1975. The formation of brine drainage features in young sea ice. J. Glaciol., 14(70), 137-154.

Eom, H.J. Unpublished. Theoretical scatter and emission models for microwave remote sensing. (Ph.D. thesis, University of Kansas, 1982.)

Evans, S. 1965. Dielectric properties of ice and snow - a review. J. Glaciol., 5(42), 773-786.

Frankenstein, G. and R. Garner. 1967. Equations for determining the brine volume of sea ice from $-0.5^{\circ}$ to $-22.9^{\circ} \mathrm{C}$. J. Glaciol., 6(48), 943-944.

Fung, A.K. and H.J. Eom. 1982. Application of a combined rough surface and volume scattering theory to sea ice and snow backscatter. IEEE Trans. Geosci. Remote Sensing, GE-20(4), 528-536.

Greely, A.W. 1886. Three years of Arctic service; an account of the Lady Franklin Bay expedition of 1881-84 and the attainment of the farthest north. London, Richard Bentley and Son.

Grenfell, T.C. 1986. Surface-based passive microwave observations of sea ice in the Bering and Greenland seas. IEEE Trans. Geosci. Remote Sensing, GE-24(3), 378-382.

Hallikainen, M., F.T. Ulaby, and M. Abdel-Razik. 1982. Measurements of the dielectric properties of snow in the $4-18 \mathrm{GHz}$ frequency range. In Proceedings, 12th European Microwave Conference. Kent, England, Microwave Exhibitions and Publishers Ltd., 151-156.

Hoekstra, P. and P. Cappillino. 1971. Dielectric properties of sea and sodium chloride ice at UHF and microwave frequencies. J. Geophys. Res., 76(20), 4922-4931.

Hulst, H.C. van de. 1957. Light scattering by small particles. New York, John Wiley and Sons.

Karam, M.A. and A.K. Fung. 1982. Propagation and scattering in multi-layered random media with rough interfaces. Electromagnetics, 2(3), 239-256.

Ketchum, R.D., jr and A.W. Lohanick. 1980. Passive microwave imagery of sea ice at $33 \mathrm{GHz}$. Remote Sensing Environ., 9(3), 211-223.

Kim Y.S. Unpublished. Theoretical and experimental study of radar backscatter from sea ice. (Ph.D. thesis, University of Kansas, 1984.)

Kim, Y.S., R.G. Onstott, and R.K. Moore. 1984. The effect of a snowcover on microwave backscatter from sea ice. IEEE J. Oceanic Eng., OE-9(5), 383-388.

Kim, Y.S., R.K. Moore, R.G. Onstott, and S. Gogineni. 1985. Towards identification of optimum radar parameters for sea-ice monitoring. J. Glaciol., 31(109), 214-219.

Lapp, D. and S. Prashker. Unpublished. Sea ice salinity and temperature data, Pond Inlet, N.W.T. Vol. 1-4. Report submitted to R.O. Ramseier, Senior Research Scientist, Atmospheric Environment Service, Downsview, Ontario, Canada.
Lohanick, A.W. and T.C. Grenfell. 1986. Variations in brightness temperature over cold first-year sea ice near Tuktoyaktuk, Northwest Territories. J. Geophys. Res., 91(C4), 5133-5144.

Malmgren, F. 1927. On the properties of sea ice. In Sverdrup, H.U., ed. The Norwegian North Polar Expedition with the Maud, 1918-1925. Scientific Results, Vol. 1a, No. 5.

Martin, S. 1979. A field study of brine drainage and oil entrainment in first-year sea ice. J. Glaciol., 22(88), 473-502.

Mätzler, C., H. Aebischer, and E. Schanda. 1984. Microwave dielectric properties of surface snow. IEEE J. Oceanic Eng., OE-9(5), 366-371.

Nelson, R.K. 1969. Hunters of the northern ice. Chicago and London, University of Chicago Press.

Ono, N. and T. Kasai. 1985. Surface layer salinity of young sea ice. Ann. Glaciol., 6, 298-299.

Onstott, R.G., R.K. Moore, S. Gogineni, and C. Delker. 1982. Four years of low-altitude sea ice broad-band backscatter measurements. IEEE J. Oceanic Eng., OE-7(1), 44-50.

Onstott, R.G., Y.S. Kim, and R.K. Moore. 1983. Preliminary report on active microwave measurements of sea ice under fall conditions. Univ. Kans. Remote Sensing Lab. Tech. Rep. 331-28/578-2.

Parashar, S.K. Unpublished. Investigation of radar discrimination of sea ice. (Ph.D. thesis, University of Kansas, 1974.)

Polder, D. and J.H. van Santen. 1946. The effective permeability of mixtures of solids. Physica, 12(5), 257-271.

Ramseier, R., P. Gloersen, W.J. Campbell, and T.C. Chang. 1975. Mesoscale description for the principal Bering Sea ice experiment. In Kondrat'yev, K. Ya., Yu. I. Rabinovich, and W. Nordberg, eds. Sovetsko-Amerikanskiy eksperiment "Bering" ... [U.S.S.R./U.S.A. Bering Sea experiment...]. Leningrad, Gidrometepizdat, 234-270.

Scott, R.F. 1905. The voyage of the "Discovery". London, Smith, Elder and Company.

Shackleton, E.H. 1909. The heart of the Antarctic. London, Heinemann.

Stiles, W.H. and F.W. Ulaby. 1980. Microwave remote sensing of snowpacks. Lawrence, KS, University of Kansas Centre for Researech, Inc. (NASA Contractor Report 3263.)

Stogryn, A. 1971. Equations for calculating the dielectric constant of saline water at $\mathrm{GHz}$ frequencies. IEEE Trans. Microwave Theory Tech., 19(8), 733.

Takizawa, T. 1985. Salination of snow on sea ice and formation of snow ice. Ann. Glaciol., 6, 309-310.

Tiuri, M.E., A.H. Sihvola, E.G. Nyfors, and M.T. Hallikainen. 1984. The complex dielectric constant of snow at microwave frequencies. IEEE J. Oceanic Eng., OE-9(5), $377-382$.

Ulaby, F.T., R.K. Moore, and A.K. Fung. 1982. Microwave remote sensing, active and passive. Vol. 2. Radar remote sensing and surface scattering and emission theory. Reading, MA, Addison Wesley.

Ulaby, F.W., W.H. Stiles, and M. Abdel-Razik. 1984. Snow cover influence on backscattering from terrain. IEEE Trans. Geosci. Remote Sensing, GE-22(2), 126-132.

Vant, M.R., R.O. Ramseier, and V. Makios. 1978. The complex dielectric constant of sea ice at frequencies in the range $0.1-40 \mathrm{GHz}$. J. Appl. Phys., 49(3), 1264-1280.

Vrangel', F.P. 1840. Narrative of an expedition to the Polar Sea, in the years 1820,1821, 1822, and 1823. London, James Madden and Company.

Wright, C.S. and R.E. Priestley. 1922. British (Terra Nova) Antarctic Expedition, 1910-1913. Glaciology. London, Harrison and Sons Ltd. 